\title{
Laser-written depressed-cladding waveguides deep inside bulk silicon
}

\author{
Ahmet Turnali, ${ }^{1}$ (i) Mertcan Han, ${ }^{1}$ and Onur Tokel ${ }^{2,3, *}$ (i) \\ ${ }^{1}$ Department of Electrical and Electronics Engineering, Bilkent University, Ankara 06800, Turkey \\ 2UNAM National Nanotechnology Research Center and Institute of Materials Science and Nanotechnology, Bilkent University, \\ Ankara 06800, Turkey \\ ${ }^{3}$ Department of Physics, Bilkent University, Ankara 06800, Turkey \\ *Corresponding author: otoke/@bilkent.edu.tr
}

Received 30 November 2018; revised 17 February 2019; accepted 18 February 2019; posted 19 February 2019 (Doc. ID 353257$)$; published 18 March 2019

\begin{abstract}
Laser-written waveguides created inside transparent materials are important components for integrated optics. Here, we demonstrate that subsurface modifications induced by nanosecond pulses can be used to fabricate tubular-shaped "in-chip" or buried waveguides inside silicon. We first demonstrate single-line modifications, which are characterized to yield a refractive index depression of $\approx 2 \times 10^{-4}$ compared to that of the unmodified crystal. Combining these in a circular geometry, we realized $2.9-\mathrm{mm}$-long, $30-\mu \mathrm{m}$ core-diameter waveguides inside the wafer. The waveguides operate in a single-mode regime at a wavelength of $1300 \mathrm{~nm}$. We use near- and far-field imaging to confirm waveguiding and for optical index characterization. The waveguide loss is estimated from scattering experiments as $2.2 \mathrm{~dB} / \mathbf{c m}$. @ 2019 Optical Society of America
\end{abstract}

https://doi.org/10.1364/JOSAB.36.000966

\section{INTRODUCTION}

Since laser-written waveguides were first demonstrated inside transparent materials two decades ago [1], they have been extensively studied in a wide array of materials, such as in glasses, polymers, and crystalline dielectrics [2-4]. Laserwritten photonic devices take advantage of the bulk of the materials, providing a significant architectural advantage, and can be fabricated using all three dimensions. Advanced control of light propagation afforded by the use of the third dimension is considered a fundamental capability for integrated optics $[5,6]$, with significant potential for electronic-photonic integration [7]. Thus, these devices have been exploited in various configurations: for instance, as optical interconnects [8]; as building blocks for more complex optical devices such as modulators, resonators and couplers [9-11]; and in diverse applications including signal processing, sensors, and imaging [12-14]. In addition, they are emerging as a platform for nonlinear dynamics and quantum photonics experiments $[15,16]$.

In spite of the interest in 3D laser-written devices in various materials, it has only recently been possible to realize fully $3 \mathrm{D}$ microstructures and buried functional optical elements in silicon [17]. These elements, which are created deep inside the wafer without altering the chip above or below the modifications are called "in-chip" devices. In-chip device fabrication is based on a form of nonlinear laser lithography, where the inherent optical response of silicon is exploited to form controlled $3 \mathrm{D}$ changes in the bulk of the wafer [17], using an extension of the nonlinear feedback-driven mechanism shown in [18]. The permanent refractive index change arises from disruptions in the crystal matrix and associated stress effects, with down to $1 \mu \mathrm{m}$ resolution [17]. The structural changes are then assembled into various three-dimensional (3D) geometries to realize functional optical devices, which include diffractive components such as holograms and gratings, as well as lenses operating at the near-infrared part of the spectrum [17].

The in-chip technology is an emerging field, where there is a significant interest to build a photonics toolbox for nearinfrared applications, including fabrication of spatial light control elements [17,19-21]. Notably, the wafer surface is unaltered after laser writing, thus the fabrication of in-chip devices leaves room for further on-chip or in-chip devices, with significant potential for multilayered functionality and novel $3 \mathrm{D}$ architectures $[17,20]$. A particularly exciting application is fabricating waveguides as a basic element of the in-chip photonics toolbox with the potential to be used in optical interconnect or sensor applications, operating at near-infrared. Similar devices have been suggested as a promising solution to active alignment and coupling problems of $\mathrm{Si}$-photonics with external optical components, for instance, interfacing with single-mode fibers [22]. In addition, they are highly desirable for integrating microfluidic technologies with photonic devices $[8,13,23]$. However, in spite of their importance for integrated optics [19] and biophotonics applications, in-chip waveguides remain limited to devices that require ultrafast lasers $[20,21]$, or are 
created with nanosecond pulses where beam propagation suffers from scattering losses as the beam propagates in the laser-modified area [19]. In fact, to the best of our knowledge, there is only one other manuscript reporting performance of an in-chip waveguide by measuring its loss coefficient [21].

In this paper, we demonstrate low-loss, depressed-index cladding in-chip waveguides fabricated with nanosecond pulses and characterize the waveguide behavior at the wavelength of $1.3 \mu \mathrm{m}$. In general, laser-written waveguides based on a refractive-index depression require a more complex 3D topology [17], compared to those based on an optical index increase [19,20]. However, the depressed-index type waveguides have an architectural advantage because the beam propagates in the unmodified core, which has well-known optical and material properties. This architecture limits losses due to absorption, allowing us to demonstrate the lowest loss in-chip waveguides.

\section{RESULTS}

In our experiments, we used the high-pulse-energy, all-fiberintegrated master-oscillator power amplifier (MOPA) system described in [24], integrated to a laser-processing station [Fig. 1(a)]. The details of the setup were reported elsewhere [17]. Briefly, we use a laser system that can produce pulses of $5 \mathrm{~ns}$ and $10 \mathrm{~W}$ power at $150 \mathrm{kHz}$ at $1.55 \mu \mathrm{m}$. The beam was directed to the processing station, where power and polarization control is achieved by a set of half-wave plates and a polarizing beam splitter. The sample position is controlled with a computer-controlled, three-axis translational stage (Aerotech, ANT130-XY, ANT95-L-Z). To evaluate the morphology and location of in-chip structures and waveguides, we used an

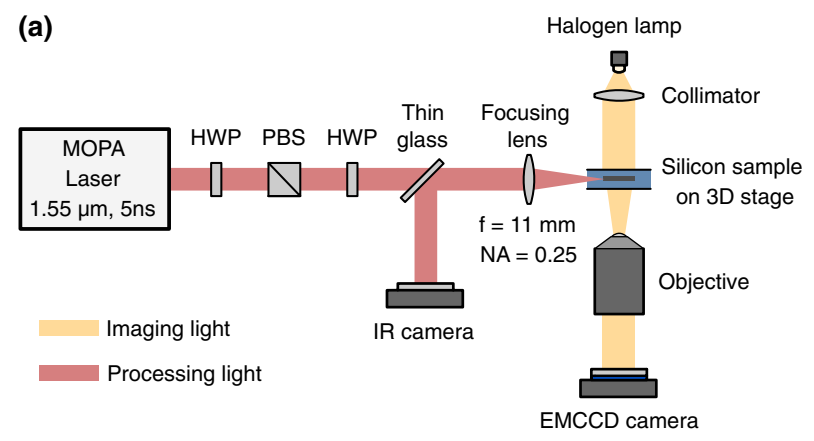

(b)

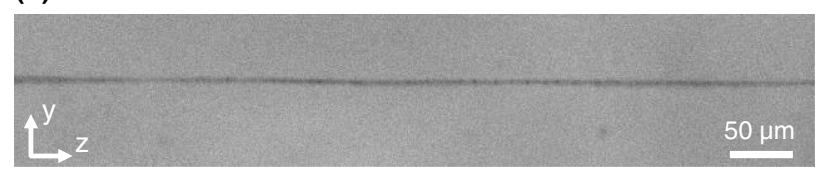

Fig. 1. (a) Pulsed laser that operates at $1.55 \mu \mathrm{m}$, along with an infrared (IR) imaging system form the experimental setup. The ns laser is used for in-chip microstructuring and photonic device fabrication, while the IR transmission microscope is used for characterization. MOPA, master-oscillator power amplifier; HWP, half-wave plate; PBS, polarizing beam splitter. (b) IR transmission microscope image of an in-chip single-line modification. The laser enters from the polished side surface $(x-y$ plane) and propagates along the $z$ axis. The sample is scanned along the optical axis to form long rod-like modifications. infrared (IR) transmission microscope, aligned perpendicular to the laser propagation direction [Fig. 1(a)].

To create in-chip microstructures, we use a longitudinal writing arrangement. The linearly polarized, collimated laser beam is focused $(f=1.1 \mathrm{~cm}, \mathrm{NA}=0.25$, aspheric lens) inside a $1 \mathrm{~mm}$ thick, $\mathrm{Si}$ wafer ( $p$-type, $\langle 100\rangle, 1 \Omega . \mathrm{cm})$ from the polished side surface. The pulse energy entering the sample is estimated as $12.6 \mu \mathrm{J}$, after accounting for surface reflection. Using these laser-writing parameters, and before scanning the laser with respect to the sample, we form $100 \mu \mathrm{m}$ long structures along the optical axis ( $z$-axis), with a measured width of $\approx 4-\mu \mathrm{m}$. These rod-like modifications are later used to form the in-chip waveguides. The structures can further be elongated by scanning the sample along the laser propagation direction [e.g., a $500 \mu \mathrm{m}$ long section is shown in Fig. 1(b)]. In this manner, we can form $2.9 \mathrm{~mm}$ long rods of uniform index change. The width remains $4 \mu \mathrm{m}$ without being significantly affected by spherical aberration due to the low numerical aperture (NA) of the lens.

We analyzed the refractive index of laser-induced structures and show that they are suitable to form waveguide claddings. For this purpose, we used an interferometric method. First, we inserted two identical unmodified silicon samples into both arms of an interferometer to form a reference pattern for the following phase-shift measurements. Then, we replaced one of the unmodified samples with a $4 \mathrm{~mm} \times 4 \mathrm{~mm} \times 0.3 \mathrm{~mm}$ volume-modified sample and obtained a second interference pattern. The refractive index change due to laser modification is calculated directly from the measured phase shift between these two patterns, using the relation $\Delta \phi=2 \pi \Delta n L / \lambda$, where $\Delta \phi$ is the measured phase shift, $\Delta n$ is the refractive index difference, $L=0.3 \mathrm{~mm}$ is the structure length along the beam propagation direction, and $\lambda$ is the wavelength. We used a home-built Raman laser as the light source, where the central wavelength $\lambda$ can be adjusted in the 1.06-1.35 $\mu \mathrm{m}$ range [25]. We characterized the optical index at $\lambda=1.30 \mu \mathrm{m}$, and found the phase shift between the reference pattern and the signal pattern as $0.14 \pi$, indicating $\Delta n$ value of $\approx 3 \times 10^{-4}$. A similar refractive index change and associated phase modulation has previously been used to demonstrate the first in-chip holograms at a different wavelength [17]. We also found, using IR microscopy, that the modifications correspond to an index depression compared to crystal Si.

We next fabricated in-chip waveguides assembled from the subsurface rod-like structures (Fig. 2). Since $\Delta n$ is negative, various $3 \mathrm{D}$ architectures are available, such as depressed cladding or parallel track configurations [3]. We chose the former, where a circularly symmetric, depressed-cladding structure encloses the higher-index waveguide core [Fig. 2(a)]. The longitudinal writing geometry minimizes asymmetry in the waveguide cross section, which could arise in transverse writing configuration due to ellipticity [6]. To fabricate circular crosssection waveguides that are $2.9 \mathrm{~mm}$ long, we took the following steps. We started by focusing the laser to a depth of $2.9 \mathrm{~mm}$ along the $z$ axis and performed a circular scan in the $x-y$ plane to form the first circular cross section. We incrementally increased the radius of the concentric circles in steps of $2 \mu \mathrm{m}$, so that the inner radius is $15 \mu \mathrm{m}$ and the outer radius is 
(a)

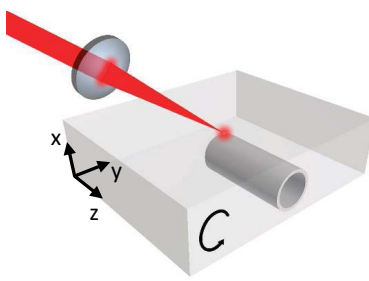

(b)

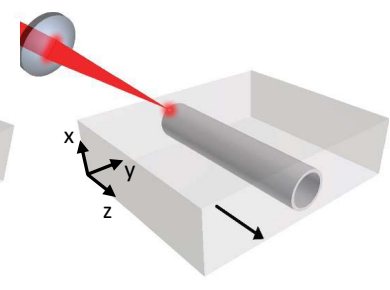

(d)

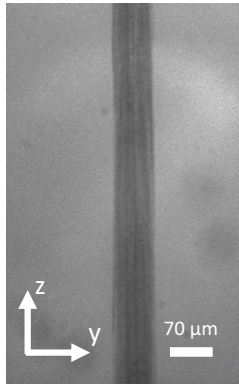

(e)

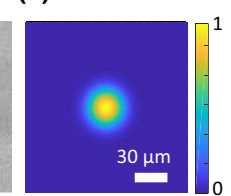

Fig. 2. (a) Illustration of the longitudinal-writing scheme to form depressed-cladding waveguides deep inside Si. We first form concentric circles at a specific depth by iteratively rotating the sample. (b) Same procedure used at different depths along $z$. (c) IR transmission microscope image showing the circular cross-section from a waveguide array, positioned $500 \mu \mathrm{m}$ from the top surface. (d) IR image of the top view of a single waveguide, where rod-like structures similar to that shown in Fig. 1(b) are used to form the cladding. (e) FDTD simulation showing the mode field profile.

$35 \mu \mathrm{m}$, forming the tubular cladding [Fig. 2(a)]. We then repeated this procedure at $100 \mu \mathrm{m}$ separated depths along the $z$ axis until we form the full waveguide [Fig. 2(b)]. The IR transmission microscope images of the waveguide cross section and top view indicate continuous and uniform modification [Figs. 2(c) and 2(d)]. The roughness (R) of the interface between the core and cladding is estimated from the standard deviation of the inner radius across the interface divided by the intended radius. $\mathrm{R}$ is found to be 0.06 , which corresponds to $\mathrm{a} \approx 1 \mu \mathrm{m}$ deviation from the intended radius. Since the laser enters from the side and can be positioned anywhere inside the wafer, we can form various 3D configurations [e.g., waveguide arrays at any depth from the top surface, as shown in Fig. 2(c)]. The mode profile of a waveguide is simulated with Lumerical [Fig. 2(e)].

To characterize these in-chip elements, we fabricated a series of waveguides in an array configuration. We coupled a $1.30 \mu \mathrm{m}$ laser beam into $2.9 \mathrm{~mm}$ long, $30 \mu \mathrm{m}$ diameter waveguides, using an aspheric lens with an NA of 0.17 . We use a low-NA lens to increase the coupling efficiency. The input and output side surfaces are polished to minimize scattering. The output light from the waveguide is directly imaged with a $20 \times$ objective (Nikon Plan Fluor $20 \times / 0.5$, WD 0.21) attached to an InGaAs camera (Artray, Artcam-031 TNIR). The near- and far-field images were acquired at the output port and $1 \mathrm{~cm}$ away, respectively. Representative far-field images for the control experiment and the waveguide output are shown in Fig. 3(a) and 3(b), respectively. A typical near-field profile from the waveguide output is shown in Fig. 3(c). The peak intensity of the output light from the waveguide output is higher than the output from bare silicon (control) as shown in Fig. 3(d), indicating waveguiding.

We estimate the numerical aperture of waveguide from independent far-field measurements, at two planes separated by $500 \mu \mathrm{m}$. We measure the divergence angle, and using the FWHM of intensity profiles, we find the NA as 0.034 .
Using the relation $\mathrm{NA} \approx(2 \mathrm{n} \Delta n)^{0.5}$, we estimate $\Delta n$ as $1.6 \times 10^{-4}$. This result is in good agreement with our previous measurement based on interference. We find the $v$ number of the waveguide as $\approx 2.4$, implying single-mode operation.

To estimate the loss coefficient $\alpha$ of waveguides, we used scattering measurements [26-28] from eight waveguides. This approach is analogous to the cutback method and assumes that the scattered light from a particular location along the waveguide is proportional to local beam intensity [26]. We used an InGaAs camera integrated with a $20 \times$ objective, recording the intensity normal to beam propagation direction in the waveguide. The measurements are performed away from the input and output ports to minimize any reflection from the end facets. As expected, we observed an exponential decrease in the scattered light intensity [Fig. 3(e)], where the slope of the fitted curve yields the loss coefficient. The loss is found in the range of $1.84-2.44 \mathrm{~dB} / \mathrm{cm}$, with a mean of $2.18 \mathrm{~dB} / \mathrm{cm}$ and standard deviation of $0.18 \mathrm{~dB} / \mathrm{cm}$. This is attributed to scattering losses from the cladding, since the guided beam propagates in the unmodified waveguide core, which has negligible absorption at this wavelength. Further, at the operated power level, nonlinear effects are negligible. The loss coefficient is the lowest reported for in-chip waveguides, and approaches the loss coefficient of analogous waveguides fabricated in silica [7]. While the longitudinal writing modality helps to decrease the ellipticity of the waveguides [Figs. 2(a) and 2(b)], we anticipate that with lower feature sizes and a smoother interface between the core and cladding, it may be possible to achieve lower loss values in future studies. We also inferred $\alpha$ by evaluating the relation, $\alpha=10 \log \left(\mathrm{P}_{0} / \mathrm{P}_{\text {meas }}\right) / L$, where $L$ is the waveguide length, $\mathrm{P}_{0}$ is power without loss, and $\mathrm{P}_{\text {meas }}$ is the power measured from waveguide output. After accounting for Fresnel reflections $(3.22 \mathrm{~dB})$, the insertion loss $(17.61 \mathrm{~dB})$, and the coupling loss $(13.98 \mathrm{~dB})$ which is calculated from the mismatch between the NA of the coupling lens and the waveguide, we estimated $\alpha=1.36-1.52 \mathrm{~dB} / \mathrm{cm}$, with a mean of $1.4 \mathrm{~dB} / \mathrm{cm}$, 

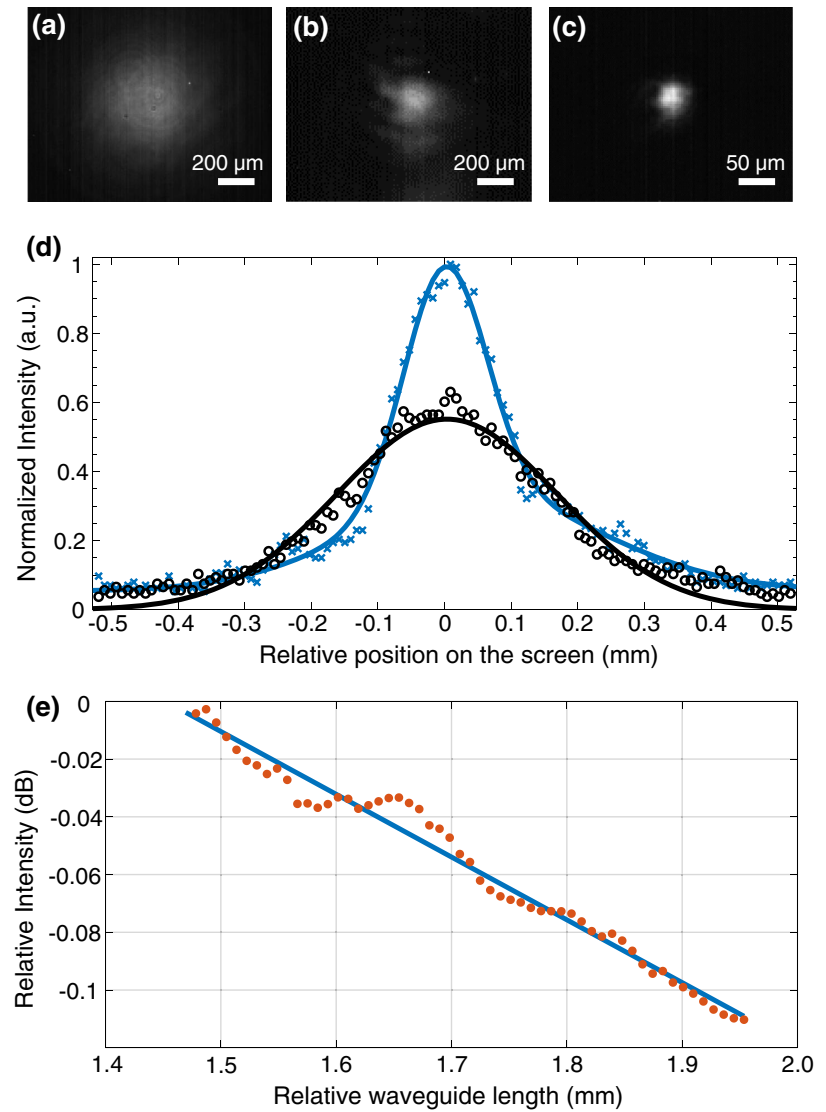

Fig. 3. (a) Far-field image of light, used as a control experiment, is acquired after passing through unmodified silicon. (b) Far-field image from the exit port of a waveguide. (c) Near-field image of the optical mode from the waveguide output port. (d) Far-field intensity profile data measured from the waveguide output (blue crosses) and the corresponding control experiment (black circles). The blue solid curve is a double Gaussian fit to the waveguide output profile. The black solid curve is a Gaussian fit to control. (e) The waveguide loss is characterized with scattering data (orange circles), filtered with a 10-pixel moving average. The blue curve shows the fitted linear curve to the logarithmic plot.

and standard deviation of $0.06 \mathrm{~dB} / \mathrm{cm}$. This result is in reasonable agreement with scattering measurements.

We finally evaluated the beam diameter and loss coefficient for different incident beam polarizations (unpolarized, circular, horizontal, and vertical polarizations). The variation of beam size between $x$ and $y$ axes is on the order of $2.5 \mu \mathrm{m}$ and is comparable to the resolving limit of the camera for near-field measurements $(2.2 \mu \mathrm{m})$. The measured diameter value and the mode profile is consistent with the corresponding single-mode beam profile obtained from simulations. Further, we observed that the measured loss coefficient for different incident beam polarizations is within experimental error of each other.

\section{CONCLUSION}

In summary, we showed that controlled laser-induced modifications inside $\mathrm{Si}$ can be used to fabricate depressed-index, tubular waveguides operating in single mode at $1.30 \mu \mathrm{m}$.
The magnitude of the refractive index change is found to be $\approx 2 \times 10^{-4}$ from measurements of waveguide NA. The waveguide loss is estimated from two measurements: power measurements as $1.4 \mathrm{~dB} / \mathrm{cm}$, and scattering experiments as $2.2 \mathrm{~dB} / \mathrm{cm}$. The waveguides can be fabricated away from the top and bottom surface and, due to the longitudinal writing geometry, do not alter the wafer surface. We demonstrated, to the best of our knowledge, the lowest-loss waveguides fabricated inside silicon, complementing the previous advances of laserwritten in-chip waveguides [19-21]. Taken together, these photonic devices can contribute to near-IR applications in integrated optics, optofluidic sensors, and Si photonics $[8,12]$.

Funding. Bilim Akademisi (BAGEP Award).

Acknowledgment. The authors kindly thank Ghaith Makey, Parviz Elahi, and F. Ömer Ilday for helpful discussions. The authors declare that there are no conflicts of interest related to this article.

\section{REFERENCES}

1. K. M. Davis, K. Miura, N. Sugimoto, and K. Hirao, "Writing waveguides in glass with a femtosecond laser," Opt. Lett. 21, 1729-1731 (1996).

2. R. R. Gattass and E. Mazur, "Femtosecond laser micromachining in transparent materials," Nat. Photonics 2, 219-225 (2008).

3. F. Chen and J. R. V. Aldana, "Optical waveguides in crystalline dielectric materials produced by femtosecond-laser micromachining," Laser Photon. Rev. 8, 251-275 (2014).

4. A. Demircan, C. Reinhardt, U. Morgner, and W. M. Pätzold, "Cascaded-focus laser writing of low-loss waveguides in polymers," Opt. Lett. 41, 1269-1272 (2016).

5. S. Nolte, M. Will, J. Burghoff, and A. Tuennermann, "Femtosecond waveguide writing: a new avenue to three-dimensional integrated optics," Appl. Phys. A 77, 109-111 (2003).

6. A. Courvoisier, M. J. Booth, and P. S. Salter, "Inscription of 3D waveguides in diamond using an ultrafast laser," Appl. Phys. Lett. 109, 031109 (2016).

7. S. J. B. Yoo, B. Guan, and R. P. Scott, "Heterogeneous 2D/3D photonic integrated microsystems," Microsyst. Nanoeng. 2, 16030-16039 (2016).

8. S. Gross and M. J. Withford, "Ultrafast-laser-inscribed 3D integrated photonics: challenges and emerging applications," Nanophotonics 4, 332-352 (2015).

9. A. Kowalevicz, V. Sharma, E. Ippen, J. Fujimoto, and K. Minoshima, "Three-dimensional photonic devices fabricated in glass by use of a femtosecond laser oscillator," Opt. Lett. 30, 1060-1062 (2005).

10. A. A. Said, G. Li, K. A. Winick, M. Dugan, and P. Bado, "Waveguide electro-optic modulator in fused silica fabricated by femtosecond laser direct writing and thermal poling," Opt. Lett. 31, 739-741 (2006).

11. G. D. Marshall, A. Politi, J. C. F. Matthews, P. Dekker, M. Ams, M. J. Withford, and J. L. O'Brien, "Laser written waveguide photonic quantum circuits," Opt. Express 17, 12546-12554 (2009).

12. R. M. Vázquez, R. Osellame, D. Nolli, C. Dongre, H. van den Vlekkert, R. Ramponi, M. Pollnau, and G. Cerullo, "Integration of femtosecond laser written optical waveguides in a lab-on-chip," Lab Chip 9, 91-96 (2009).

13. Y. Bellouard, A. Champion, B. Lenssen, M. Matteucci, A. Schaap, M. Beresna, C. Corbari, M. Gecevicius, P. Kazansky, O. Chappuis, M. Kral, R. Clavel, F. Barrot, J. M. Breguet, Y. Mabillard, S. Bottinelli, M. Hopper, C. Hoenninger, E. Mottay, and J. Lopez, "The Femtoprint project," J. Laser Micro/Nanoeng. 7, 1-10 (2012).

14. S. Weimann, A. Perez-Leija, M. Lebugle, R. Keil, M. Tichy, M. Gräfe, R. Heilmann, S. Nolte, H. Moya-Cessa, G. Weihs, D. N. Christodoulides, and A. Szameit, "Implementation of quantum and 
classical discrete fractional Fourier transforms," Nat. Commun. 7, 11027 (2016).

15. M. Heinrich, R. Keil, F. Dreisow, A. Tunnermann, A. Szameit, and S. Nolte, "Nonlinear discrete optics in femtosecond laser-written photonic lattices," Appl. Phys. B 104, 469-480 (2011).

16. T. Meany, M. Gräfe, R. Heilmann, A. P. Leija, S. Gross, M. J. Steel, M. J. Withford, and A. Szameit, "Laser written circuits for quantum photonics," Laser Photon. Rev. 9, 363-384 (2015).

17. O. Tokel, A. Turnalı, G. Makey, P. Elahi, T. Çolakoğlu, E. Ergecen, Ö. Yavuz, R. Hübner, M. Z. Borra, I. Pavlov, A. Bek, R. Turan, D. K. Kesim, S. Tozburun, S. Ilday, and F. Ö. Ilday, "In-chip microstructures and photonic devices fabricated by nonlinear laser lithography deep inside silicon," Nat. Photonics 11, 639-645 (2017).

18. B. Öktem, I. Pavlov, S. Ilday, H. Kalaycıoğlu, A. Rybak, S. Yavaş, M. Erdoğan, and F. Ö. Ilday, "Nonlinear laser lithography for indefinitely large-area nanostructuring with femtosecond pulses," Nat. Photonics 7, 897-901 (2013).

19. M. Chambonneau, Q. Li, M. Chanal, N. Sanner, and D. Grojo, "Writing waveguides inside monolithic crystalline silicon with nanosecond laser pulses," Opt. Lett. 41, 4875-4878 (2016).

20. I. Pavlov, O. Tokel, S. Pavlova, V. Kadan, G. Makey, A. Turnali, Ö. Yavuz, and F. Ö. Ilday, "Femtosecond laser written waveguides deep inside silicon," Opt. Lett. 42, 3028-3031 (2017).

21. G. Matthäus, H. Kämmer, K. A. Lammers, C. Vetter, W. Watanabe, and S. Nolte, "Inscription of silicon waveguides using picosecond pulses," Opt. Express 26, 24089-24097 (2018).
22. H. Terasawa, F. Tan, O. Sugihara, A. Kawasaki, D. Inoue, T. Yamashita, M. Kagami, O. Maury, Y. Bretonniere, and C. Andraud, "Light-induced self-written waveguide fabrication using $1550 \mathrm{~nm}$ laser light," Opt. Lett. 42, 2236-2238 (2017).

23. A. Schaap, Y. Bellouard, and T. Rohrlack, "Optofluidic lab-on-a-chip for rapid algae population screening," Biomedical Opt. Express 2, 658-664 (2011).

24. I. Pavlov, E. Dülgergil, E. Ilbey, and F. Ö. Ilday, "Diffraction-limited, 10-W, 5-ns, 100-kHz, all-fiber laserat 1.55 нm,"Opt. Lett. 39, 2695-2698(2014).

25. P. Elahi, G. Makey, A. Turnali, O. Tokel, and F. Ö. Ilday, "1.06 $\mu \mathrm{m}-$ $1.35 \mu \mathrm{m}$ coherent pulse generation by a synchronously-pumped phosphosilicate Raman fiber laser," in European Conference on Lasers and Electro-Optics and European Quantum Electronics Conference (2017), paper CJ21.

26. S. V. Rao, K. Moutzouris, M. Ebrahimzadeh, A. De Rossi, G. Gintz, M. Calligaro, V. Ortiz, and V. Berger, "Influence of scattering and twophoton absorption on the optical loss in $\mathrm{GaAs}-\mathrm{Al}_{2} \mathrm{O}_{3}$ nonlinear waveguides measured using femtosecond pulses," IEEE J. Quantum Electron. 39, 478-486 (2003).

27. S. M. Eaton, H. Zhang, P. R. Herman, F. Yoshino, L. Shah, J. Bovatsek, and A. Y. Arai, "Heat accumulation effects in femtosecond laser-written waveguides with variable repetition rate," Opt. Express 13, 4708-4716 (2005).

28. J. Xia, A. M. Rossi, and T. E. Murphy, "Laser-written nanoporous silicon ridge waveguide for highly sensitive optical sensors," Opt. Lett. 37, 256-258 (2012). 